\title{
Drawing physical insight from mathematics via extreme case reasoning
}

\author{
Mark Eichenlaub ${ }^{1}$, Deborah Hemingway ${ }^{2}$, and Edward F. Redish ${ }^{1}$ \\ ${ }^{1}$ Department of Physics, University of Maryland, College Park, MD, 20742-4111 \\ ${ }^{2}$ Biophysics Program, University of Maryland, College Park, MD, 20742-2431
}

\begin{abstract}
Students often get stuck in problem solving, ignoring their physical intuition in favor of plug-and-chug or pattern-matching approaches. We suggest that examining the extreme cases is a useful way of moving students towards more expert-like problem solving. Based on a case study, we show that novice students can quickly learn to use extreme cases productively in problem-solving. In reasoning about extreme cases, students blend conceptual and mathematical cognitive resources. At the same time, they can generate new and creative uses for extreme-case reasoning, here recasting it from a tool for evaluating answers to one for generating them. Extreme case reasoning may prove a valuable instructional goal at the introductory level.
\end{abstract}

\section{INTRODUCTION}

We are interest in how students learn to make physical meaning of math during problem-solving. Most studies on this topic have looked at how students translate physical situations into equations and how they later interpret the equations' solutions. The intermediate step of how students manipulate equations remains relatively unexplored as a source of physical insight. Frameworks for analyzing mathematical problem solving, for example the ACER framework of Wilcox et al. [1] or the epistemic games of Tuminaro and Redish [2], often assign sense-making and mathematical manipulations completely separate steps in the problem-solving process. Experts, however, do find the individual mathematical manipulations to be physically meaningful in some cases, as exemplified by Kustusch et al. [3] in a study of physics professors taking physically-motivated partial derivatives in solving a thermodynamics problem. Although the manipulation step is important to expert sensemaking, Kuo et. al. reviewed the problem-solving literature on this topic, finding "no studies that focused upon the mathematical processing step in quantitative problem solving or described alternatives to using equations as computational tools." [4] They argued that in the manipulation step, students can not only bridge between math and physics, but blend them into a cohesive cognitive structure. There is little research on how to encourage this type of blended mathematical sense-making in courses. Here, we suggest that when students push the variables in a problem to their extremes, they access new resources which enrich their process of manipulating equations, including blending intuitive and mathematical thinking in creative ways.

As part of an ongoing project to study student use of mathematics in introductory physics for the life sciences, we identified four patterns of inquiry, called epistemic games (egames) [2], that we expected students to learn to use productively over the course of a year-long physics sequence. Our goal was to investigate how students can use these e-games to deepen the interplay between their formal mathematical knowledge and physical intuition in the problem-solving process. Those games were dimensional analysis, examine extreme cases, estimation, and build maps from symbols to physical meaning. We then conducted problem-solving interviews to learn about the cognitive and epistemological resources students use when they play these e-games. Here, we analyze video data on the case study of Bert, a student who was encouraged to play the examine extreme cases game when solving a mechanics problem and later played the game productively in the context of a geometry problem that he had previously been unable to solve.

Bert not only adopted the extreme cases game readily, he used it in new and creative ways. Although the game was presented to him as a tool to test and distinguish between potential answers that have been generated by other means, he used it to place constraints on what sort of symbolic form [5] the correct, unknown answer should have, recasting the game from a tool for evaluating equations to one for generating new ones, blending mathematical formalism with physical intuition in the process. We suggest that further work on examining extreme cases may turn it into a useful instructional tools for encouraging expert-like mathematical sense-making.

\section{BACKGROUND}

A student solving a generic problem can potentially call on any piece of knowledge, type of reasoning, or physical tool at their disposal. Most of these will be irrelevant, so to narrow the possibilities, they first decide (often unconsciously) what type of situation they are in. This process, called "framing", reduces the possibility space people search through [6, 7]. Framing a situation often cues a script for how that type of activity typically goes, which sets expectations for what will happen next and what sorts of actions are appropriate [8]. In problem solving, students often follow a class of scripts called epistemic games [2].

In an epistemic game, as in games like solitaire or chess, one or several players make moves. These moves might be mathematical moves, such as add the same quantity to both sides of the equation, conversational moves, such as offer a reason supporting your position, or physical moves, such as draw a picture of the situation. As the players make moves, they gradually fill out an epistemological form, a template 
for what the solution to the problem should look like, which may be physical or verbal. Finally, players either reach the e-game's stopping condition and decide they are done, or else switch to a different game or give up on the solution attempt.

Because students' choices of e-game can restrict their access to productive resources [2,9], it's possible that identify-ing and teaching new e-games will help students use more expert-like problem solving tools. We chose to study the extreme case e-game because it is familiar to expert prob-lem solving in physics and mathematics, and often cited as a method of building intuition $[10,11]$.

The game begins after a student completes the mathematical manipulation stage of solving a problem and wants to evaluate the equation they've obtained as a solution to the original problem. The steps we preliminarily propose for this e-game, based on our experience rather than on analyz-ing data, are

1. Identify a physical variable in the problem.

2. Imagine it becoming extremely large or small.

3. Intuitively identify the behavior of the system.

4. Analyze the same limit of the potential solution.

5. Compare the results of (3) and (4) for consistency.

6. Repeat for other variables in the problem.

What is interesting here is the epistemic role this game plays in expert problem solving. It is a tool that only partially confirms a potential solution. For example, a physics PhD student solving a mechanics problem on the question-and-answer website Quora wrote in his response to a mechanics question [12]

This one gets pretty ugly. I did it with Lagrangian mechanics, and got $F_{N}=\frac{(M+m) g}{1+(m / M) \sin ^{2} \theta}$, but I did it a bit quickly so I can't vouch for the answer (although it does look like it has the right behavior in the $m \ll M$ and $m \gg M$ limits).

If a solution matches intuition in the extreme cases, this evidently doesn't prove it to be correct from this expert's point of view, but does lend the solution some additional credence. Previous work on extreme cases suggests that even young students can use them to activate new and productive conceptual resources [13], and that the mechanism for this likely relates to extreme cases being powerful ways to generate dynamic mental imagery [14].

\section{METHODOLOGY}

Our study is part of a project to understand use of mathematics in physics class in the NEXUS/Physics introductory physics for the life sciences course [15]. The students were mostly juniors and had previously taken courses in calculus and several science courses. Most were majoring in biology or related fields and many are pre-medical or pre-health students.

Our report is on preliminary research into student use of extreme cases. Our goal was to generate hypotheses regarding

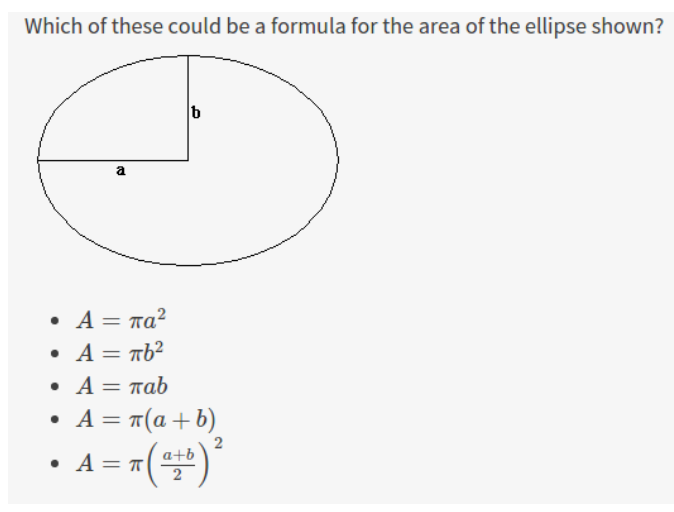

FIG. 1. The area of an ellipse problem

how students adopt, use, and adapt extreme case reasoning. Future research will attempt to analyze these generated hypotheses in detail. We began studying the extreme cases with the expectation that most students would not have it readily available as a problem-solving strategy, and so sought to study it in an interview environment where the interviewer could scaffold the process to varying degrees during problem solving in response to the student's thinking. To that end, we conducted six one-hour video-recorded individual interviews with students using a think-aloud protocol [16].

We then reviewed the interviews to select incidents that stood out as surprising in how students proceeded, rich in the variety of reasoning resources used, or clear in the type of thinking students were employing. The researchers agreed that one interview with Bert (a pseudonym), a male student from the class, stood out on all three fronts, and so we analyze that interview in detail here.

In this interview, Bert was first asked to solve the geometry problem on the area of an ellipse (see fig. 1). In ten minutes, he was unable to decide on the correct answer. The interviewer then gave Bert a problem on the acceleration of a half-Atwood machine (see fig. 2). After Bert reached an answer to this problem, the interviewer introduced the extreme cases e-game, and Bert realized that his answer was not correct and worked towards a new solution. We then returned to the ellipse problem, where Bert spontaneously applied extreme case reasoning and found the correct answer.

\section{RESULTS AND DISCUSSION}

The half-Atwood machine is a traditional physics problem which we expected interviewees to solve via standard epistemic games. When Bert first approached this problem, he played the game physical mechanism [2], whose epistemic form does not involve equations.

21:41 Bert: But if there's no friction then it should just slide along the table with ease with no negative force acting on it, so if I'm pulling downwards on this block, that's also pulling sideways with the same force. So then if this 


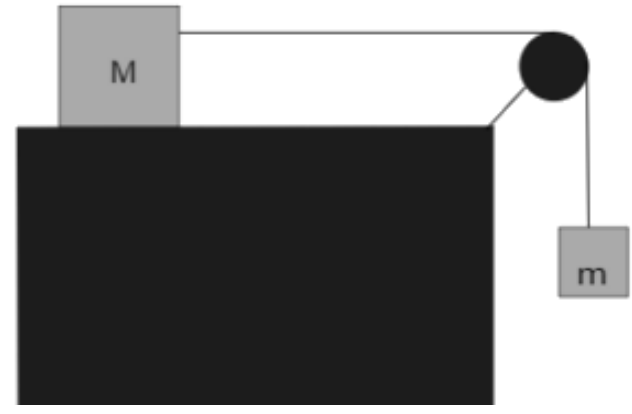

FIG. 2. The half-atwood problem

Problem text: "A block of mass $M$ is attached to a block of mass $m$ via a massless string strung over a pulley as show. The setup is frictionless. What is the acceleration of the block $m$ ?"

is in that doesn't have any resistive force then I guess the little m would just be. What's the question? What's the acceleration? Would be negative nine point eight meters per second squared. That's gravity. And that would be my answer.

Bert proceeds directly from a physical story about the situation - that the block is pulled by gravity and that there is no resistance - to an incorrect conclusion, that the block accelerates with local gravitational acceleration. The interviewer then makes two bids to reconsider the completeness of this story, one qualitative and one quantitative.

22:18 I: So what's gonna happen to... just kind of like tell me roughly what's going to happen to both of these blocks.

22:23 Bert: They're both gonna move at the same increasing speed. Block little $\mathrm{m}$ will move down block big $m$ will move to the right.

22:31 I: Okay. And what would happen if I made this little block say half as big? How would that change the motion?

22:40 Bert: Um, it wouldn't because gravity is independent on mass. No it's not. Yes it is. Yeah. Gravity is independent on mass

Bert demonstrates some uncertainty when asked about cutting the mass a block in half, but quickly rejects the idea that his answer needed to be revised.

However, when the interviewer asks about the extreme case, Bert changes his method of evaluating his answer, initially holds to his original solution, then waivers, then goes back to trying to solve the problem

22:59 I: Okay. Um, what if this one weighed a million pounds and this one weighed one ounce?

23:06 Bert: Um, there's no friction though, so still I'm thinking it would somehow move it, as weird as that sounds, because like, kind of like, if you were up in space and you tap the block that weighs a billion pounds it's still gonna move.

In thinking about an extreme case, Bert's epistemological stance has changed. Whereas he previously appeared to be engaged in recall ("Does gravity depend on mass?"), he now discusses how an idea is "as weird as it sounds", an indicator that he is engaged more in physical intuition.

Bert goes on to identify tension in the rope as an important factor in the problem which he had previously omitted, draws a free body diagram, and obtains an answer that is correct apart from a sign error. However, once he has solved the equations he set up, Bert doesn't attempt to interpret his new solution. Instead, he stops and waits for the interviewer to decide what to do next. The interviewer introduces the game check extreme cases by scaffolding each step of the process.

33:33 I: So what happens to the formula if this mass becomes really really big and this mass becomes really really small?

33:41 Bert: Um, then this would be the large negative number on bottom and on the top would be small so acceleration [draws and immediately erases some sort of fraction] would be low in the negative direction. Would be low acceleration.

33:59 I: Okay so in the negative direction. So which direction is negative here?

\section{4:04 Bert: Down}

34:05 I: Okay, so it would move downward?

34:08 Bert: Yeah, slowly.

34:10 I: What happens if we reverse it? What happens if we make this mass, little $\mathrm{m}$, be really, really heavy?

34:19 Bert: So then it doesn't make sense because then it's in the positive direction because...

In playing check extreme cases, Bert discovers errors he pre-viously didn't, quickly following the interviewer's prompts and making productive use of the extreme cases egame. Bert and the interviewer continue checking extreme cases. It appears Bert is not yet ready or expecting to play the game autonomously in the interview setting. He often stops, wait-ing for the interviewer to introduce the next move, and even-tually says he is getting frustrated and erases all his work. Before reworking the entire problem, the interviewer asks whether Bert can make a modification to his existing solu-tion so that it works in the extreme cases. Bert begins mak-ing various guesses and testing them, for example insert-ing an absolute value in the denominator to prevent it from changing sign, a problem he had noticed with its behavior. Amid these informed guesses, Bert says 
40:04 Bert: I dunno ahh... I made it. I want it... try to reverse it to $M$ minus $m$ [erases numerator of previous equation and changes to $M-m$ ] So then this is super big that's super small. [pauses, draws a minus sign on the numerator] Still doesn't make sense. Still not working. Cause one of these are big then it's gonna be big acceleration. That's not what should happen. Should be as this one grows [points to $M$ ] it gets smaller, so like that has to be in the denominator.

In this passage, Bert adopts the extreme cases game as his own. He plays the game without interviewer intervention, in contrast to ten minutes previously. Additionally, Bert notes that the intuitive requirement that the acceleration decreases with increasing mass of the top block means that $M$ must go in the denominator of his equation for acceleration. He is using what Sherin [5] identified as a symbolic form, a relation between a template for an equation, in this case a division template $(\ldots / \square)$ with the conceptual pattern of inverse one variable going up and another going down. This sort of mixed mathematical-intuitive sensemaking was proposed as a worthy instructional target by Kuo et. al [4].

In several more minutes of evaluating potential solutions, Bert never considers the correct answer, and the interviewer suggests returning to the ellipse problem, which Bert had examined earlier in the interview, but been unable to decide whether the area of an ellipse was $\pi a b$ or $\pi\left(\frac{a+b}{2}\right)^{2}$. On looking back at the ellipse problem, Bert draws an ellipse approximately ten times as wide as its height, commenting "So I'm just going to do some extreme examples ... they're easier to see. I do extreme examples sometimes. So kind of like the problem we just did we were looking at extreme examples."

Bert not only transfers the extreme cases game to a new context, he takes ownership of it as something "I do ... sometimes". Playing this game, Bert examines the extreme cases of both his possible equations, and is able to identify only $\pi a b$ as behaving correctly in the limit of a long, skinny ellipse.

\section{v. CONCLUSIONS}

Expert physics problem solvers move fluidly between intuition and formal mathematics, often combining them into blended cognitive structures. Although novices sometimes do the same [4], the phenomenon is little-studied in physics education research, and is rarely an instructional goal. When it is an instructional goal, it can be difficult to design systems that reward students for expert-like mathematical sense-making without making it just another checkbox on a rubric [17]. In the example of Bert, we see that guiding students through epistemic games such as examine extreme cases can cause significant shifts to students' epistemic frames and lead to new, creative sorts of blended mathematical and physical cognition. Additionally, Bert was able to pick up on the game quickly and successfully apply it to problems he had previously failed to solve. This suggests that the extreme cases game and similar epistemic games have the potential to be useful tools for guiding students towards problem solving expertise.

\section{ACKNOWLEDGMENTS}

Thank you to the entire Maryland Physics Education Research Group for commenting on this data, and to Janet Walkoe for extensive comments on an earlier version of this work. This material is based upon work supported by the US National Science Foundation under Award No. 15-04366.
[1] B. R. Wilcox, M. D. Caballero, D. A. Rehn, and S. J. Pollock, Physical Review Special Topics - Physics Education Research 9 (2013), ISSN 15549178, 1307.0791.

[2] J. Tuminaro and E. F. Redish, Physical Review Special Topics-Physics Education Research 3, 020101 (2007).

[3] M. B. Kustusch, D. Roundy, T. Dray, and C. A. Manogue, Physical Review Special Topics - Physics Education Research 10, 1 (2014), ISSN 15549178.

[4] E. Kuo, M. M. Hull, A. Gupta, and A. Elby, Science Education 97, 32 (2013).

[5] B. L. Sherin, Cognition and instruction 19, 479 (2001).

[6] T. J. Bing and E. F. Redish, Physical Review Special TopicsPhysics Education Research 5, 020108 (2009).

[7] D. Hammer, A. Elby, R. E. Scherr, and E. F. Redish, Transfer of learning from a modern multidisciplinary perspective pp. 89-120 (2005).

[8] J. R. Anderson, Cognitive psychology and its implications (Macmillan, 2005).
[9] T. J. Bing and E. F. Redish, Physical Review Special TopicsPhysics Education Research 8, 010105 (2012).

[10] G. Polya, How to solve it: A new aspect of mathematical method (Princeton university press, 2014).

[11] J. C. Nearing et al., Mathematical tools for physics (Dover Publications, 2010).

[12] E. Anson, If a body $(m)$ slides down a frictionless inclined plane (2015), [Online; accessed 14-December-2015], URL http://qr.ae/1At $4 \mathrm{eF}$.

[13] A. Zietsman and J. Clement, The Journal of the Learning Sciences 6, 61 (1997).

[14] J. J. Clement and L. Stephens, in Proceedings of the 2009 Annual Meeting of the National Association for Research in Science Learning (2009).

[15] E. Redish, C. Bauer, K. Carleton, T. Cooke, M. Cooper, C. H. Crouch, B. Dreyfus, B. Geller, J. Giannini, J. S. Gouvea, et al., American Journal of Physics 82, 368 (2014).

[16] D. N. Perkins and D. N. Perkins, The mind's best work (Harvard University Press, 2009).

[17] M. M. Hull, E. Kuo, A. Gupta, and A. Elby, Physical Review Special Topics-Physics Education Research 9, 010105 (2013). 